\title{
Structure et dynamique des systèmes désordonnés
}

\author{
P. Damay
}

LASIR, CNRS, 13 rue de Toul, 59046 Lille cedex, France

\section{résumé}

Définis par leur « absence » d'ordre, les systèmes désordonnés ne forment pas à proprement parler une famille. La notion de désordre est elle-même assez difficile à définir. Aussi, dans ce type d'étude, le type de systèmes, les techniques et les concepts sont-ils particulièrement diversifiés.

Après avoir énoncé quelques grandes généralités sur la définition du désordre et fait une tentative de classification des systèmes désordonnés, cette revue a été principalement orientée sur les moyens d'études de la structure et de la dynamique des fluides et des verres. Une insistance particulière est mise sur les moyens mis à disposition par la physique statistique aussi bien pour élaborer des modèles que pour analyser les résultats d'expériences spectroscopiques ou de diffraction. En effet, le nombre de configurations locales des atomes et molécules dans ces systèmes est tellement important que les résultats expérimentaux et les modèles ne décrivent en général que des moyennes d'ensemble qui n'apportent que des informations partielles sur la réalité microscopique.

\section{ORDRE ET DESORDRE}

\subsection{La notion de désordre}

La notion de désordre se définit comme une déviation par rapport à l'image que l'on peut avoir d'un état ordonné de la matière.

On peut se faire une image assez simpliste de la notion d'ordre à l'aide de quelques exemples. Pour bâtir un système ordonné, il faut se définir une règle. Dans le cas des cristaux, le concept fondamental est celui du réseau de Bravais. La règle est la suivante : à partir d'un noeud pris comme origine, on peut atteindre n'importe quel point du réseau par la translation

$$
\mathbf{R}=n_{1} \mathbf{a}_{1}+n_{2} \mathbf{a}_{2}+n_{3} \mathbf{a}_{3}
$$

-en positionnant les atomes sur chaque site, on obtient un cristal parfait ordonné.

-de même dans un système binaire $\mathrm{AB}$, par exemple un cristal de $\mathrm{NaCl}$, on obtient un état ordonné si tous les ions se trouvent sur des sites cristallins bien définis et si chaque ion $\mathrm{Na}^{+}$est entouré par un même nombre d'ions $\mathrm{Cl}$ et réciproquement.

-en positionnant des molécules sur les sites cristallins, on définit un cristal moléculaire ordonné. La définition d'ordre orientationnel s'ajoute alors à celle d'ordre translationnel. 
À partir de ces exemples, on imagine assez facilement plusieurs façons d'introduire du désordre dans ces structures idéales :

désordre thermique. Sous l'effet de la température, les atomes acquièrent une petite énergie cinétique qui leur permet de se déplacer légèrement autour de leur position cristalline idéale. Dans une expérience de diffraction de RX ou de neutrons, on observe alors une diminution de l'intensité des pics de Bragg, surtout aux grandes valeurs de transfert de moment. L'intensité perdue se retrouve alors dans un bruit de fond diffus.

désordre chimique. Dans le système binaire $\mathrm{AB}$, des atomes $\mathrm{A}$ et $\mathrm{B}$ échangent leurs positions.

défauts et impuretés. Un désordre est introduit si l'on enlève des atomes de certains sites, si l'on en ajoute sur quelques sites interstitiels ou si un certain nombre d'atomes est remplacé par des atomes d'une autre espèce.

désordre orientationnel. Les molécules d'un cristal moléculaire peuvent être positionnées exactement sur le réseau cristallin, mais prendre des orientations désordonnées. De même, dans les cristaux magnétiques, les atomes sont situés sur des sites cristallins, mais les spins peuvent être plus ou moins alignés.

De ces exemples simples, il apparait que la définition de l'ordre ou du désordre pour un même système peut ne pas être unique. Le système peut posséder un ordre parfait de position mais pas d'orientation. En fait l'ordre sera défini par une règle [ordering rule] pour chaque propriété, par exemple ordre de position, ordre chimique, ordre orientationnel, ordre magnétique...

Il existe des méthodes beaucoup plus expéditives pour introduire du désordre dans un cristal parfait ; on peut l'irradier fortement, implanter des ions, le faire fondre, le vaporiser...A l'état de vapeur dans un grand volume, les atomes sont très éloignés les uns des autres et n'interagissent plus entre eux, l'énergie cinétique moyenne de chaque atome est égale à $3 / 2 \mathrm{kT}$, ce qui définit le gaz parfait. Si le cristal parfait à température nulle peut paraître comme la référence de l'ordre de position, le gaz parfait sert de référence au désordre complet.

\subsection{Le paramètre d'ordre}

Clairement, il existe une grande différence de nature du désordre entre le cristal dont $99 \%$ des atomes sont en position ordonnée et le gaz parfait qui ne présente aucun caractère ordonné. Dans la littérature, on peut relever des expressions telles que «partiellement ordonné », « vestige de l'ordre cristallin »... Il est besoin d'introduire un paramètre quantitatif pour caractériser l'ordre ou le désordre, c'est le « paramètre d'ordre ». La notion de paramètre d'ordre a été le plus fréquemment utilisée pour discuter des transitions de phases avec variation de l'ordre (transitions ordre-désordre), mais elle reste parfaitement adaptée pour caractériser l'ordre dans chacune des phases. 
Deux exemples simples permettent de préciser le concept de paramètre d'ordre :

- $\quad$ Magnétisation $\mathbf{M}$ d'une assemblée de spins. Dans l'exemple choisi, les spins peuvent s'orienter « up » ou « down » comme dans le ferromagnétisme. La magnétisation à saturation $\mathbf{M}_{\text {sat }}$ correspond à un alignement de tous les spins dans la même direction. Des spins orientés aléatoirement donnent une magnétisation nulle $\mathbf{M}=0$. La magnétisation est ainsi un bon paramètre d'ordre capable de caractériser les états dans lesquels l'orientation est partielle.

Alliage binaire $A B_{n}$. Soit $c_{a}$ la fraction molaire d'atomes de l'espèce $A$ et $c_{B}=I$ $c_{A}$ la fraction molaire d'atome $B$ dans l'échantillon. Si $r$ est la fraction d'atomes de l'espèce $A$ se trouvant en position ordonnée ( comme dans le cristal parfait), le paramètre d'ordre se définit par

$$
P=\frac{r-c_{A}}{1-c_{A}}=\frac{r-c_{A}}{c_{B}}
$$

De fait $P$ est égal à 1 pour le cristal parfait où l'ordre chimique l'emporte et $P$ est égal zéro quand la position des atomes est au hasard $\left(r=c_{A}\right)$

La notion de paramètre d'ordre donne ainsi un sens à l'expression «partiellement ordonné ".

Il est cependant risqué de chercher une définition générale de la notion de paramètre d'ordre. A chaque propriété et chaque « ordering rule » correspond un paramètre d'ordre le mieux adapté. Dans les deux exemples choisis, le paramètre d'ordre est un scalaire, mais c'est en général une quantité beaucoup plus complexe ; il peut posséder plusieurs dimensions, être scalaire, vecteur ou tenseur. Une recherche bibliographique sur « order parameter » retourne plus de 25000 entrées. Quelques autres exemples seront montrés dans les paragraphes suivants.

\section{L' ETUDE DES SYSTEMES DESORDONNES}

\subsection{Les grandes familles de systèmes désordonnés}

En s'appuyant essentiellement sur les propriétés structurales, les systèmes désordonnés ou partiellement ordonnés peuvent se classer en quatre familles:

1- les systèmes désordonnés qui ne possèdent pas d'ordre à longue distance ; la diffraction de RX ou neutrons ne présente pas de pics de Bragg. Dans cette famille, on classera les gaz, les liquides et solutions, les polymères, les verres et autres solides amorphes.

2- les cristaux désordonnés. Le lien qui relie les membres de cette famille est l'existence d'un ordre à longue distance donnant lieu à l'apparition de pics de Bragg dans la figure de diffraction. Dans de nombreux systèmes, le paramètre d'ordre est proche de l'unité et le désordre peut être traité comme une perturbation. Il s'agit du désordre thermique qui diminue l'intensité des pics de Bragg, du désordre causé par les impuretés, le dopage ou les 
dislocations...Le désordre est plus important dans les alliages chimiquement désordonnés. Il s'agit de cristaux formés par exemple de deux types d'atomes de dimensions semblables. Si les interactions chimiques sont faibles, les atomes peuvent être distribués aléatoirement sur les sites cristallins.

3- les cristaux liquides, nématiques, smectiques et cholestériques. Formés le plus souvent de longues molécules polaires ou non, les cristaux liquides possèdent un ordre orientationnel qui peut être à très longue portée et un ordre de position de portée limitée.

4- les phases plastiques. Il s'agit de cristaux moléculaires, molécules dont les centres de masse sont situés sur des sites cristallin. L'orientation des groupements moléculaires est désordonnée.

Les cristaux liquides et phases plastiques pourraient être classés respectivement dans les systèmes désordonnés et les cristaux désordonnés mais leurs spécificités et leur importance peut justifier la distinction.

Dans ces familles, les systèmes peuvent être plus ou moins désordonnés. Par exemple, l'orientation des molécules dans les cristaux liquides peut être plus ou moins prononcée ou le désordre chimique dans les alliages plus ou moins complet. Le choix judicieux du paramètre d'ordre permettra de caractériser les états d'ordre partiel. De plus, d'autres états désordonnés comme les quasi-cristaux, les gels, aérogels ou autres fractals, les composites, les super réseaux entrent difficilement dans cette classification.

\subsection{Difficultés particulières}

L'étude des systèmes désordonnés pose toujours des problèmes difficiles à résoudre. S'il est relativement aisé de définir une règle pour définir l'ordre (exemple équation1.1), il est plus difficile de trouver les outils mathématiques pour caractériser les structures désordonnées.

Dans un cristal, les informations données par la détermination de la cellule unitaire qui ne contient en général qu'un ou quelques atomes est suffisante pour connâitre la structure du cristal macroscopique.

Dans un système désordonné comme un liquide, le nombre de configurations que peut prendre l'environnement de chaque particule est considérable. Il faudrait déterminer la position de chacune des particules pour connaître la structure; même s'il était possible de faire l'expérience, le résultat serait inexploitable. Inévitablement, il faut utiliser les outils de la mécanique statistique pour faire des moyennes. Ainsi on déterminera la distance moyenne d'un atome avec les proches voisins ainsi que la dispersion de distances ; la notion de proche voisin se prêtant elle-même à discussion.

De plus les particules dans un fluide sont en mouvement permanent et les configurations changent continuellement. La dynamique devra toujours être prise en compte dans le calcul des moyennes. 
Beaucoup de systèmes désordonnés, comme les verres et les semi-conducteurs dopés, ne sont pas à l'équilibre thermodynamique. Il ne suffit pas, dans ces cas de connaître la composition chimique, la température et la pression pour définir le système, des variables comme l'histoire, la méthode de préparation, les traitements thermiques peuvent jouer un rôle considérable dans l'établissement de la structure.

Enfin, les expériences ne donnent le plus souvent que des informations partielles sur la structure. Par exemple, si la distance moyenne entre les particules peut être obtenue avec une bonne précision, les informations sur l'arrangement spatial ne sont obtenues que de manière indirecte. Typiquement, les expériences de diffraction ( $\mathrm{RX}$ ou neutrons) ne donnent des informations que dans une dimension (fonction de distribution radiale).

\section{STRUCTURE DES SYSTEMES DESORDONNES}

\subsection{Une approche topologique du désordre}

Comment appréhender la «structure » d'un système désordonné si on abandonne l'idée de déterminer les coordonnées de chaque atome ? Une approche plus modeste mais plus efficace est d'évaluer la distribution des distances interatomiques et le nombre moyen de plus proches voisins autour d'un atome de référence.

Soit un système fluide monoatomique; les atomes sont considérés comme des sphères de rayon $\sigma$. Le potentiel de Lennard-Jones est un bon modèle pour caractériser l'interaction entre deux atomes dans un fluide monoatomique :

$$
V(r)=4 \varepsilon\left[\left(\frac{\sigma}{r}\right)^{12}-\left(\frac{\sigma}{r}\right)^{6}\right]
$$

Le premier terme définit la répulsion à courte distance des orbitales due au principe d'exclusion de Pauli ; le second terme est un terme attractif à plus longue portée de type Van der Waals. A la température $T$, l'énergie cinétique d'origine thermique $3 / 2 \mathrm{k}_{\mathrm{B}} \mathrm{T}$ se superpose au potentiel. Si l'énergie thermique est très faible devant la profondeur $\varepsilon$ du puits, les atomes premiers voisins chercheront une position d'équilibre à une distance $r_{e}$ proche du diamètre $2 \sigma$ de chaque atome.

Comment évaluer le nombre moyen de plus proches voisins ?

$\mathrm{Au}$ point triple, on remarque que généralement la fusion s'accompagne d'une expansion de volume qui reste modérée $(\approx 15 \%)$, une indication que la compacité et par conséquent le nombre de proches voisins n'est que peu affectée par la transition ordre-désordre en passant du solide cristallin au liquide.

Dans les systèmes cristallins compacts tels que les systèmes cubique face centrée(FCC) et hexagonal compact $(\mathrm{CPH})$, chaque atome est entouré et en contact avec douze atomes également en contact. Dans ces systèmes qui sont les plus compacts, les atomes, supposés 
sphériques, occupent 74\% de l'espace (soit une compacité $\eta$ de 0.74 ). Dans le cristal les 12 atomes ne sont pas uniformément répartis sur la sphères de rayon $2 \sigma$ autour de l'atome origine (la position compacte des atomes forme 8 sites tétraédriques et 6 sites octaédriques ).

Dans un empilement désordonné compact $(k T<<\varepsilon)$, en absence de forces orientationnelles, on recherche une distribution plus homogène sur la sphère ; la solution est de positionner les atomes sur les sommets d'un icosaèdre régulier. Les atomes ne sont alors en contact qu'avec l'atome central et forment sur la sphère des arrangements pentagonaux. Il n'est pas possible de continuer l'empilement icosaédrique compact pour obtenir l'ordre à longue distance ; mais les critères d'isotropie orientationnelle locale et l'existence de symétrie icosaédrique à longue distance dans les quasi-cristaux suggèrent que l'ordre icosaédrique à courte distance dans les systèmes désordonnés tels que les liquides ou les verres est possible et même vraisemblable.

Il est apparu clairement que l'empilement compact de sphères désordonnées était un bon départ pour modéliser les liquides et verres monoatomiques. Mais comment reconstruire l'empilement désordonné à grande échelle à partir d'un germe tel que l'icosaèdre, par exemple ? Il n'existe malheureusement pas de modèle mathématique pour empiler les sphères.

Bernal a étudié les configurations des sphères empilées dans une boîte ; la boîte est agitée afin d'obtenir la compacité maximale. La compacité maximale jamais observée est $\eta=0.64$; le nombre moyen de proches voisins est de 8.5 et les arrangements pentagonaux des voisins sont majoritaires.

Voronoi a utilisé le concept de la cellule de Wigner-Seitz dans les cristaux (ou zone de Brillouin dans le réseau réciproque ) pour tracer des polyèdres définis par les plans bissecteurs entre l'atome de référence et ses voisins ; ainsi le volume de la cellule comprend la portion de l'espace située le plus près de l'atome inclus. Si la zone de Wigner-Seitz est unique dans le cristal, on peut dénombrer quelques dizaines de polyèdres dans un système désordonné simulé sur ordinateur. Pour des sphères identiques, la simulation sur ordinateur retrouve une compacité maximale de 0.64 .

Une idée plus récente introduite par Bernal a été de considérer la configuration des sites interstitiels. Les polyèdres de Voronoi sont construits autour des sites interstitiels au lieu des atomes. Dans les cristaux compacts FCC ou HCP, les sites sont des tétraèdres ou des octaèdres. On retrouve ces mêmes sites mais déformés dans le système désordonné mais aussi des sites de volume plus important (dodécaèdres...). Cette approche permet également d'aborder des systèmes formés de sphères de tailles différentes.

L'approche topologique est très enrichissante car ce type de modélisation permet de visualiser les différents arrangements locaux qui se retrouvent vraisemblablement dans les systèmes réels ; de plus, elle montre la grande complexité et multiplicité de solutions. Néanmoins cette démarche reste surtout dans le domaine de la visualisation et l'utilisation des fonctions de distribution définies dans le cadre de la physique statistique s'avère indispensable pour dépasser l'ordre purement local et s'occuper des systèmes réels macroscopiques. 


\subsection{Les fonctions de distribution}

\subsubsection{Les systèmes monoatomiques}

Soit un fluide désordonné (gaz ou liquide) constitué de $N$ atomes dans un volume $V$. La densité moyenne, ou nombre d'atomes par unité de volume s'écrit

$$
\rho_{0}=N / V
$$

La fonction de distribution à une particule $\rho^{(1)}(\mathbf{r})$ peut s'écrire en utilisant la fonction $\delta$ :

$$
\rho^{(1)}(\mathbf{r})=\sum_{i=1}^{N} \delta\left(\mathbf{r}-\mathbf{r}_{i}\right)
$$

où $\mathbf{r}_{i}$ est la position du centre de masse de chaque atome. Comme les atomes ne sont pas fixes, $\rho^{(1)}(\mathbf{r})$ est une moyenne sur le temps de la fonction-densité dépendant du temps $\rho^{(1)}(\mathbf{r}, t)$;

$$
\rho^{(1)}(\mathbf{r}, t)=\sum_{i=1}^{N} \delta\left(\mathbf{r}-\mathbf{r}_{i}(t)\right)
$$

où $\mathbf{r}_{\mathrm{i}}(t)$ représente la position instantanée de l'atome $\mathrm{i}$.

Ainsi $\quad \rho^{(1)}(\mathbf{r})=\int \rho^{(1)}(\mathbf{r}, t) d t$

L'intégrale de $\rho^{(1)}(\mathbf{r})$ sur le volume redonne le nombre de particules

$$
\int_{V} \rho^{(1)}(\mathbf{r}) d \mathbf{r}=N
$$

Rappelons que dr représente un élément de volume égal à $d x . d y \cdot d z$ dans un repère cartésien et égal à $r^{2} \sin \theta d r d \theta d \varphi$ en coordonnées sphériques.

La fonction densité de paires $\rho^{(2)}\left(\mathbf{r}_{1}, \mathbf{r}_{2}\right)$ s'écrit de la même façon

$$
\rho^{(2)}\left(\mathbf{r}_{1}, \mathbf{r}_{2}\right)=\sum_{i=1}^{N} \sum_{j \neq i}^{N-1} \delta\left(\mathbf{r}_{1}-\mathbf{r}_{i}\right) \delta\left(\mathbf{r}_{2}-\mathbf{r}_{j}\right)
$$

Cette fonction est la moyenne sur le temps de la fonction densité de paires dynamique :

$$
\rho^{(2)}\left(\mathbf{r}_{1}, \mathbf{r}_{2}\right)=\int \rho^{(2)}\left(\mathbf{r}_{1}, \mathbf{r}_{2}, t\right) d t
$$

en moyenne sur le temps, la fonction $\rho^{(2)}\left(\mathbf{r}_{1}, \mathbf{r}_{2}\right)$ n'est pas nulle que si l'on trouve à la fois un atome en $\mathbf{r}_{i}$ et un atome en $\mathbf{r}_{j}$.

Ainsi $\rho^{(2)}\left(\mathbf{r}_{1}, \mathbf{r}_{2}\right) d \mathbf{r}_{1} d \mathbf{r}_{2}$ est la probabilité de trouver en même temps un atome dans les micro volumes $d \mathbf{r}_{1}$ et $d \mathbf{r}_{2}$. 
L'intégrale sur le volume $d \mathbf{r}_{2}$ donne une relation entre la fonction à deux particules et la fonction à une particule :

$$
\int_{V} \rho^{(2)}\left(\mathbf{r}_{1}, \mathbf{r}_{2}\right) d \mathbf{r}_{2}=(N-1) \rho^{(1)}\left(\mathbf{r}_{1}\right)
$$

La probabilité de trouver un atome en $d \mathbf{r}_{2}$ peut dépendre de la probabilité d'occupation de $d \mathbf{r}_{1}$ si les deux volumes ne sont pas trop éloignés, ainsi :

$$
\rho^{(2)}\left(\mathbf{r}_{1}, \mathbf{r}_{2}\right) \neq \rho^{(1)}\left(\mathbf{r}_{1}\right) \rho^{(1)}\left(\mathbf{r}_{2}\right)
$$

Cette inégalité permet de définir la fonction de distribution de paires $g^{(2)}\left(\mathbf{r}_{1}, \mathbf{r}_{2}\right)$

$$
\rho^{(2)}\left(\mathbf{r}_{1}, \mathbf{r}_{2}\right)=\rho^{(1)}\left(\mathbf{r}_{1}\right) \rho^{(1)}\left(\mathbf{r}_{2}\right) g^{(2)}\left(\mathbf{r}_{1}, \mathbf{r}_{2}\right)
$$

Si le système est homogène, n'importe quel point de l'espace peut être pris comme origine ; de plus $\rho^{(1)}\left(\mathbf{r}_{1}\right)=\rho^{(1)}\left(\mathbf{r}_{2}\right)=\rho_{0}$. Dans ce cas, on peut remplacer $\mathbf{r}_{1}, \mathbf{r}_{2}$ par $\mathbf{r}=\mathbf{r}_{2}-\mathbf{r}_{1}$.

$$
\rho^{(2)}\left(\mathbf{r}_{1}, \mathbf{r}_{2}\right)=\rho_{0}^{2} g^{(2)}\left(\mathbf{r}_{1}, \mathbf{r}_{2}\right)=\rho_{0}^{2} g^{(2)}(\mathbf{r})
$$

Pour les systèmes isotropes (liquides, verres), la moyenne sur toutes les orientations de $\mathbf{r}$ donne

$$
g(r)=g^{(2)}(|\mathbf{r}|)
$$

où l'exposant (2) est omis par commodité.

La figure 1 représente l'allure typique de la fonction $g(r)$ pour un liquide monoatomique. La fonction est nulle jusqu'à la distance de moindre approche, on observe un premier pic correspondant à la première sphère de coordination puis des oscillations de moins en moins définies lorsque la distance augmente.

La figure montre bien la portée des interactions dans un fluide. Le premier pic intense et étroit correspond à la localisation des proches voisins, pratiquement en contact avec l'atome d'origine ; la faible largeur du pic indique que la distribution des distances est faible autour de la distance la plus probable. Les oscillations qui suivent correspondent aux couches successives, l'intensité diminue et la largeur augmente considérablement. La corrélation disparaît aux grandes distances. Dans des fluides très structurés comme les sels fondus, on peut observer jusqu'à 10 couches ; on en observe beaucoup moins dans les fluides de Van der Waals ( 3 à 4 ). 


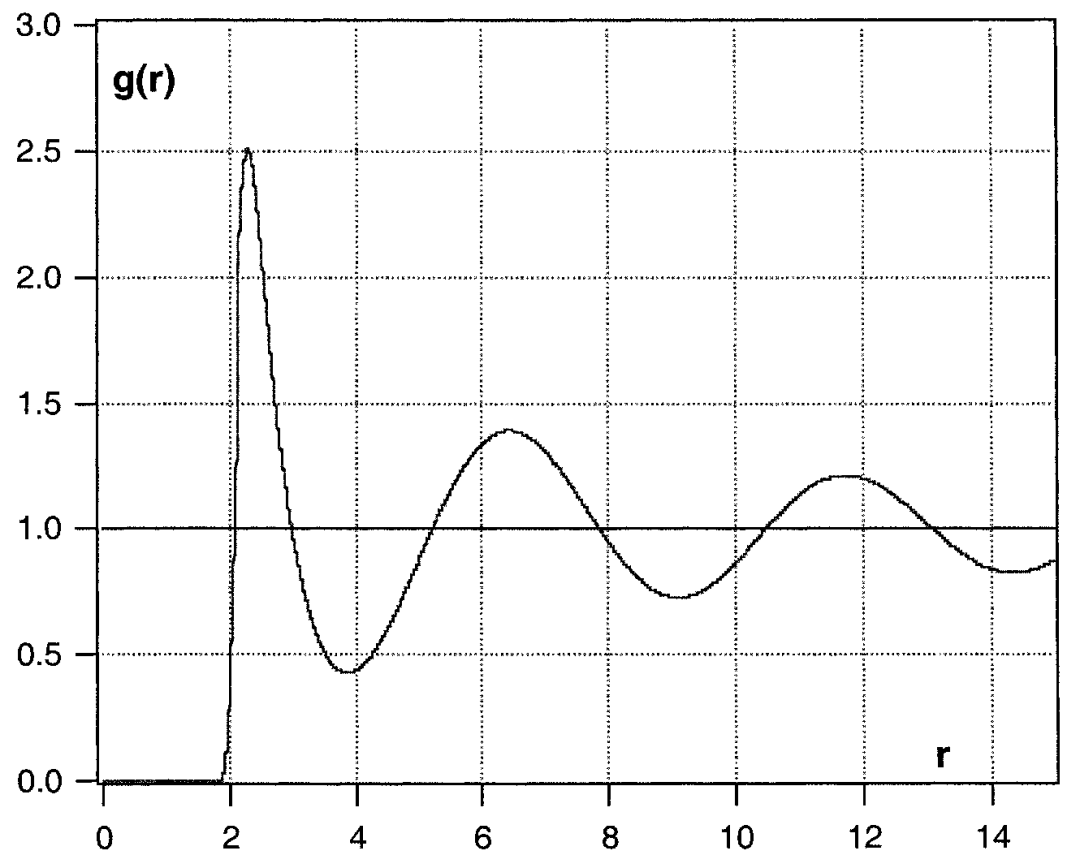

Figure l . fonction de distribution de paire $\mathrm{g}(\mathbf{r})$ pour un fluide monoatomique.

La fonction de distribution de paires $g(r)$ caractérise les distances entre atomes. Une fonction d'ordre supérieur comme $\rho^{(3)}\left(\mathbf{r}_{1}, \mathbf{r}_{2}, \mathbf{r}_{3}\right)=\rho_{0}^{3} g^{(3)}\left(\mathbf{r}_{1}, \mathbf{r}_{2}, \mathbf{r}_{3}\right)$ décrivant la probabilité de trouver en même temps une particule dans $d \mathbf{r}_{1}$, dans $d \mathbf{r}_{2}$ et dans $d \mathbf{r}_{3}$ donne les premières informations directes sur l'arrangement local impliquant les orientations.

L'intégrale sur $d \mathbf{r}_{3}$ est égale à

$$
\int_{V} \rho^{(3)}\left(\mathbf{r}_{1}, \mathbf{r}_{2}, \mathbf{r}_{3}\right) d \mathbf{r}_{3}=(N-2) \rho^{(2)}\left(\mathbf{r}_{1}, \mathbf{r}_{2}\right)
$$

indiquant qu'il existe un très grand nombre de possibilités d'arranger trois atomes pour la même fonction de distribution de paires. Cette observation est importante car elle souligne combien les informations procurées par la détermination de la fonction de distribution de paires $g(r)$ sont limitées. Malheureusement, les expériences de diffraction sur les systèmes désordonnés ne fournissent le plus souvent que la distribution de paires.

Le nombre d'atomes situés dans une coquille sphérique à une distance comprise entre $r$ et $r+d r$ à l'origine est égal à 


$$
n=\rho_{0} g(r) d \mathbf{r}=4 \pi r^{2} \rho_{0} g(r) d r
$$

ce qui définit la fonction de distribution radiale

$$
R D F(r)=4 \pi r^{2} \rho_{0} g(r)
$$

En particulier, le nombre de coordination, nombre de plus proches voisin de l'atome situé à l'origine est égal à

$$
n_{\text {coor }}=\int_{0}^{r_{1}} R D F(r) d r
$$

où $r_{l}$ est choisi de façon à intégrer la surface sous le premier pic de $g(r)$.

On note que le préfacteur dans la fonction densité de paire $\rho^{(2)}\left(\mathbf{r}_{1}, \mathbf{r}_{2}\right)$ (eq. 3.12) est égal à $\rho_{0}^{2}$ alors qu'il égal à $\rho_{0}$ dans la définition de la RDF. La fonction densité de paires est égale à la probabilité $\rho_{0}$ de trouver un atome à l'origine multiplié par la probabilité $\rho_{0} g(r)$ de trouver un atome à la distance $r$ alors que dans la définition de la RDF, on suppose qu'il y a un atome à l'origine (probabilité=1).

On définit également la fonction de corrélation de paires $G(r)$ qui fait ressortir l'écart au désordre complet

$$
G(r)=4 \pi \rho_{0} r[g(r)-1]
$$

Le facteur de structure $S(Q)$ est obtenu par transformée de Fourier de la fonction de distribution radiale

$$
S(Q)=1+\rho_{0} \int_{0}^{\infty}[g(r)-1] e^{i \mathbf{Q} \cdot \mathbf{r}} d \mathbf{r}
$$

Pour un système homogène et isotrope, la moyenne sur toutes les orientations de $\left\langle e^{i \mathbf{a} \cdot r}\right\rangle$ est égale à $\sin Q r / Q r$ et $d \mathbf{r}=4 \pi r^{2} d r$; ainsi

$$
S(Q)=1+\rho_{0} \int_{0}^{\infty}[g(r)-1] \frac{\sin Q r}{Q r} 4 \pi r^{2} d r
$$

Le facteur de structure $S(Q)$ est la grandeur mesurée dans une expérience de diffusion de neutrons, par exemple. L'échantillon est irradié par un faisceau monochromatique de neutrons de longueur d'onde $\lambda$. L'expérience consiste à mesurer l'intensité diffusée en fonction de l'angle $\theta$. Le vecteur de transfert de moment est défini $\operatorname{par} Q=\frac{4 \pi}{\lambda} \sin \theta$. En se rappelant la définition de $g(r)$, la diffraction sur les liquides est une moyenne de photos instantanées de la structure du fluide.

La section efficace différentielle $d \sigma / d \Omega$ mesurée au cours d'une expérience de diffusion de neutrons est proportionnelle au carré de la longueur de diffusion cohérente multiplié par le facteur de structure, plus la diffusion incohérente indépendante de l'angle (voir le cours de Jacques Schweizer):

$$
\frac{1}{N} \frac{d \sigma}{d \Omega}=b_{c o h}^{2} S(Q)+b_{i n c}^{2}
$$


où $\mathrm{N}$ est le nombre d'atomes irradiés, $\sigma$ la section efficace de diffusion et $d \Omega=\sin \theta d \theta d \varphi$ est l'élément différentiel de l'angle solide

La fonction de distribution de paires peut être déterminée par transformée de Fourier inverse à partir de $S(Q)$ mesuré :

$$
g(r)=1+\frac{1}{2 \pi^{2} r \rho_{0}} \int_{0}^{\infty}[S(Q)-1] \sin Q r d Q
$$

\subsubsection{Les systèmes polyatomiques}

Dans les systèmes polyatomiques, par exemple un alliage liquide binaire constitué de $\mathrm{m}$ atomes de l'espèce $\mathrm{A}$ et $\mathrm{n}$ atomes de l'espèce $\mathrm{B}$, il est nécessaire de différentier l'atome choisi pour origine. Il faut rechercher la distribution d'atomes $A$ autour d'un atome $A$ pris comme origine, puis la distribution des atomes $\mathrm{B}$ autour de ce même atome $\mathrm{A}$ et ainsi de suite; ce qui définit les fonctions de distribution de paires partielles $g_{A A}(r), g_{B B}(r)$ et $g_{A B}(r)$ avec $g_{A B}(r)=g_{B A}(r)$. La fonction de distribution totale est égale à la somme des fonctions de distributions partielles pondérées par les longueurs de diffusion et les concentrations de chaque espèce. Pour l'alliage binaire $A_{m} B_{n}$, la fonction de distribution totale s'écrit

$$
g(r)=\frac{c_{A}^{2} b_{A}^{2} g_{A A}(r)+2 c_{A} c_{b} b_{A} b_{B} g_{A B}(r)+c_{B}^{2} b_{B}^{2} g_{B B}(r)}{\left(c_{A} b_{A}+c_{B} b_{B}\right)^{2}}
$$

avec $c_{A}=m /(m+n)$ et $c_{B}=n /(m+n)$ les fractions molaires de $\mathrm{A}$ et $\mathrm{B}$ et $b_{A}, b_{B}$ les longueurs de diffusion cohérente pour les deux types d'atomes. Le dénominateur assure la normalisation.

L'extension de l'équation 3.22 à des systèmes comprenant plus d'atomes est immédiate ; pour $\mathrm{n}$ types d'atomes différents, il existe $\mathrm{n}(\mathrm{n}+1) / 2$ fonctions de distributions partielles.

A chaque fonction de distribution partielle correspond un facteur de structure partiel obtenu par transformée de Fourier :

$$
S_{i j}(Q)=1+\rho_{0} \int_{0}^{\infty}\left[g_{i j}(r)-1\right] \frac{\sin Q r}{Q r} 4 \pi r^{2} d r
$$

où $\rho_{0}$ est la densité d'atomes, c'est-à-dire $\sum_{i} n_{i} \times \frac{1}{V}$

Le facteur de structure total déterminé à partir d'une expérience de diffusion de neutrons est la combinaison des facteurs de structure partiels pondérés par la longueur de diffusion et la concentration de chaque type d'atome; la méthode de substitution isotopique permet, dans les mélanges simples, de déterminer l'environnement de chaque type d'atome.

A la différence des systèmes monoatomiques, on note que le facteur de structure $S(Q)$ total mesuré par diffusion de neutrons ou de RX pour un système polyatomique ne représente pas 
une propriété intrinsèque du système mais dépend de la technique utilisée. Ce sont les facteurs de structure partiels $S_{i j}(Q)$ qui sont indépendants de la sonde de mesure.

\section{DESORDRE ET DYNAMIQUE}

\subsection{Déplacements moléculaires dans les systèmes désordonnés}

Le mouvement des atomes est plus facile à comprendre dans les gaz ou les cristaux parfaits que dans les liquides ou les amorphes.

Dans un gaz, les atomes se déplacent linéairement avec une vitesse définie par la statistique de Boltzmann. Le libre parcourt moyen $\ell=\langle v\rangle \tau$ avec $\tau$ le temps moyen entre deux collisions.

Dans les cristaux, on s'attend à ce que les déplacements d'atomes restent localisés (oscillations autour de la position d'équilibre). Les mouvements peuvent cependant être collectifs et donner par exemple naissance à la propagation des phonons. La fréquence de propagation $\omega$ dépend du vecteur d'onde $\mathbf{k}$. Les informations sont données par la courbe de dispersion $\omega(\mathbf{k})$. Comme dans le cas de la détermination de la structure, grâce à la périodicité, il suffit de déterminer les courbes de dispersion $\omega(\mathbf{k})$ à l'intérieur de la zone de Brillouin (branche acoustique et branche optique) pour caractériser les mouvements collectifs.

La dynamique des systèmes désordonnés est souvent très complexe ; par exemple dans les liquides, les vibrations des molécules correspondent à des mouvements atomiques individuels, les rotations sont corrélées dans beaucoup de cas, les atomes ou molécules peuvent se déplacer par diffusion et les ondes acoustiques, mouvements collectifs de grande longueur d'onde se déplacent avec des vitesses dépendant de la densité et la compressibilité. Les mouvements collectifs de faible longueur d'onde sont amortis et difficiles à mettre en évidence.

\subsection{La fonction de corrélation de Van Hove}

Dans le paragraphe précédent, on s'est intéressé à positionner les atomes dans un système désordonné fluide ou amorphe. Dans un liquide, les atomes sont en perpétuel mouvement ; la structure est une moyenne sur le temps de toutes les configurations; mais une description plus complète du fluide requiert la prise en compte des aspects dynamiques. Il est ainsi nécessaire de définir une fonction de distribution plus générale incluant la position et le moment des atomes ; cette fonction peut s'écrire $f\left(\mathbf{r}_{1}, \mathbf{p}_{1}, t_{1} ; \mathbf{r}_{0}, \mathbf{p}_{0}, t_{0}\right)$, donnant la probabilité de trouver un atome de moment $\mathbf{p}_{1}$ en $\mathbf{r}_{1}$ au temps $\mathbf{t}_{1}$ s'il y avait un atome de moment $\mathbf{p}_{0}$ en $\mathbf{r}_{0}$ au temps $t_{0}$.

La plupart des propriétés dynamiques peuvent être décrites avec une expression plus simple $G(\mathbf{r}, t)$, fonction de l'espace et du temps qui est obtenue en effectuant la moyenne sur les moments. Si le système est isotrope, homogène et à l'équilibre thermodynamique, seuls la 
distance $\mathbf{r}=\mathbf{r}_{1}-\mathbf{r}_{0}$ entre les atomes et l'intervalle de temps $t=t_{1}-t_{0}$ sont des variables pertinentes. Ainsi

$$
G(\mathbf{r}, t)=\iint f\left(\mathbf{r}_{1}, \mathbf{p}_{1}, t_{1} ; \mathbf{r}_{0}, \mathbf{p}_{0}, t_{0}\right) d \mathbf{p}_{1} d \mathbf{p}_{0}
$$

Cette relation est la fonction de corrélation de Van Hove.

D'un point vue mathématique, la probabilité de trouver un atome $j$ en $\mathbf{r}^{\prime}$ au temps $t$ est égale à $\delta\left[\mathbf{r}^{\prime}-\mathbf{r}_{j}(t)\right]$, celle de trouver l'atome $\mathbf{i}$ au temps $\mathrm{t}=0$ en $\mathbf{r}^{\prime}-\mathbf{r}$ est $\delta\left[\mathbf{r}-\mathbf{r}^{\prime}+\mathbf{r}_{i}(0)\right] . \mathrm{La}$ probabilité de trouver les deux évènements est

$$
\delta\left[r-r^{\prime}+r_{i}(0)\right] d\left[r^{\prime}-r_{j}(t)\right]
$$

Il convient d'intégrer sur $d \mathbf{r}^{\prime}$ puisque l'origine peut être prise n'importe où dans le fluide, puis d'effectuer la somme sur toutes les paires $i j$ et enfin de prendre la moyenne thermique ; la fonction de Van Hove s'écrit alors

$$
G(\mathbf{r}, t)=\frac{1}{N}\left\langle\sum_{i, j} \int_{V} \delta\left[\mathbf{r}-\mathbf{r}^{\prime}+\mathbf{r}_{i}(0)\right] \cdot \delta\left[\mathbf{r}^{\prime}-\mathbf{r}_{j}(t)\right] d \mathbf{r}^{\prime}\right\rangle
$$

L'ordre des sommations n'intervient pas dans les systèmes classiques et

$$
G(\mathbf{r}, t)=\frac{1}{N}\left\langle\sum_{i, j} \delta\left[\mathbf{r}+\mathbf{r}_{i}(0)-\mathbf{r}_{j}(t)\right]\right\rangle
$$

La fonction de corrélation de Van Hove peut s'exprimer à partir de la fonction-densité dynamique (éq. 3.4) $\rho(\mathbf{r}, t)$ :

$$
G(\mathbf{r}, t)=\frac{1}{N} \int_{V}\left\langle\rho\left(\mathbf{r}-\mathbf{r}^{\prime}, 0\right) \rho\left(\mathbf{r}^{\prime}, t\right)\right\rangle d \mathbf{r}^{\prime}
$$

Dans l'équation 4.4, il convient de distinguer le cas ou $i=j$ qui caractérise l'auto-corrélation, décrivant la position de l'atome au cours du temps (par exemple l'auto diffusion) et le cas $i \neq j$ caractérisant les mouvements corrélés d'atomes ; soit $G_{s}(\mathbf{r}, t)$ et $G_{d}(\mathbf{r}, t), s$ pour 'self' et $d$ pour 'distinct'. Par exemple, $G_{s}(\mathbf{r}, 0)$ est nulle pour tout $\mathbf{r} \neq 0$ car l'atome ne peut pas se trouver à deux endroits au même instant.

La fonction de corrélation est reliée à $g(r)$ pour $t=t_{1}-t_{0}=0$, en effet

$$
G(\mathbf{r}, 0)=G_{s}(\mathbf{r}, 0)+G_{d}(\mathbf{r}, 0)=\delta(r)+\rho g(r)
$$

La double transformée de Fourier de $G(r, t)$ est le facteur de structure dynamique $S(Q, \omega)$ qui peut être mesuré par exemple par diffusion inélastique des neutrons; la simple transformée de Fourier $r \Rightarrow Q$ donne la fonction de diffusion intermédiaire $S(Q, t)$. 


\section{METHODES ET MOYENS}

Une étude précise des propriétés des systèmes désordonnés se heurte à des difficultés considérables. C'est la conjonction de plusieurs techniques expérimentales et théoriques qui permet de proposer des modèles microscopiques vraisemblables et efficaces.

Les mesures thermodynamiques et l'utilisation de toute la machinerie de la physique statistique sont à développer en premier lieu. Le nombre de configurations, c'est-à-dire la multiplicité d'arrangements est la principale caractéristique des systèmes désordonnés. L'entropie $S=k_{B} l n W$, où $W$ est le nombre de configurations, est une mesure directe de l'importance du nombre de configurations. La détermination de la chaleur spécifique $C_{V}=T\left(\frac{\partial S}{\partial T}\right)_{V}$ donne directement la variation du nombre de configurations avec la température. De même, la mesure des propriétés de transport dans les liquides apporte des informations macroscopiques essentielles à la compréhension de propriétés dynamiques. Puisque le nombre de configurations locales est très important et qu'il est illusoire de faire une description exhaustive de ces arrangements, l'utilisation de tous les moyens de la physique statistique est fondamentale. L'approche statistique permet d'effectuer les moyennes appropriées sur les propriétés physiques, moyennes qui sont susceptibles d'être mesurées ; un exemple est la fonction de distribution de paires qui peut être obtenue à partir d'une mesure de diffraction de RX ou de neutrons.

Le cas des verres et amorphes, ou des liquides surfondus qui ne sont pas à l'équilibre thermodynamique, posent des problèmes délicats qu'il faut aborder avec soin. Néanmoins, comme beaucoup de mouvements locaux restent actifs dans ces systèmes et que l'évolution vers l'état thermodynamique stable peut être très lente, il est souvent possible d'appliquer des formules statistiques sur ces systèmes.

Enfin il faut insister sur l'importance grandissante que prennent les simulations ou 'expériences sur ordinateur' dans l'étude des systèmes désordonnés. A partir de modèles d'interactions microscopiques bien choisis, les méthodes telles que la dynamique moléculaire ou simulation par méthode de Monte Carlo sont devenues plus que des compléments aux mesures expérimentales. Les propriétés thermodynamiques, propriétés de transport et facteurs de structures pour des systèmes de plus en plus complexes peuvent maintenant être retrouvées avec une précision suffisante pour valider le choix des potentiels d'interaction. Par exemple près d'une publication sur deux traitant de la détermination du facteur de structure de liquides accompagne maintenant les résultats de simulation numérique. 


\section{Références}

The physics of Structurally Disordered Matter ; an Introduction

N.E.Cusack

IOP publishing, Bristol 1987

Theory of Neutron Scattering from Condensed Matter

S.W.Lovesey

Clarendon, Oxford 1984

Bernal J.D. Nature 183,141 (1959)

Introduction to Phase Transitions and Critical Phenomena

H.E. Stanley

Clarendon Press. Oxford 1971

Proceedings of the fifth International conference on the Structure on Non-Crystalline Materials (NCM5)

Sendaii, Japan, 1991

K.Suzuki, A.C.Wright, editors

North-Holland, , Amsterdam, 1992

Liquides, cristallisation et transition vitreuse

Les Houches 1989

Editeurs J.P.Hansen, D.Levesque et J.Zinn-Justin

North-Holland, Amsterdam, 1991

Neutron and Synchrotron Radiation for Condensed Matter Studies

Volume 1 : Theory, Instruments and Methods

J.Baruchel, J.L.Hodeau, M.S.Lehmann, J.R.Regnard, C.Schlenker, Editors

Springer-Verlag, Berlin, 1993.

Les Editions de Physique, Les Ulis, 1993.

An Introduction to the Liquid State

P.A. Egelstaff

Clarendon Press, Oxford 1994

Theory of Simple Liquids

J.P. Hansen and I.R. McDonald

Academic Press, London 1976 